\title{
Controlled Formation of Closed-Edge Nanopores
}

\section{in Graphene}

Kuang $\mathrm{He}^{1}$, Alex W. Robertson ${ }^{1}$, Chuncheng Gong ${ }^{1}$, Christopher S. Allen ${ }^{1}$, Qiang $\mathrm{Xu}^{2}$, Henny Zandbergen ${ }^{3}$, Angus I. Kirkland ${ }^{1}$, Jamie H. Warner ${ }^{1 *}$

${ }^{1}$ Department of Materials, University of Oxford, Parks Road, Oxford, OX1 3PH, United Kingdom

${ }^{2}$ DENSsolutions, Delftechpark 26, 2628 XH Delft, The Netherlands. ${ }^{3}$ Kavli Institute of Nanoscience, Delft University of Technology, Lorentzweg 1, 2628 CJ Delft, The Netherlands.

* Jamie.Warner@materials.ox.ac.uk

\section{Received Date}

\begin{abstract}
:
Dangling bonds at the edge of a nanopore in monolayer graphene make it susceptible to back-filling at low temperatures from atmospheric hydrocarbons, leading to potential instability for nanopore applications, such as DNA sequencing. We show that closed edge nanopores in bilayer graphene are robust to back-filling under atmospheric conditions for days. A controlled method for closed edge nanopore formation starting from monolayer graphene is reported using an in-situ heating holder and electron beam irradiation within an aberration-corrected transmission electron microscopy. Tailoring of closed-edge nanopore sizes is demonstrated from $1.4-7.4 \mathrm{~nm}$. These results should provide mechanisms for improving the stability of nanopores in graphene for a wide range of applications involving mass transport.
\end{abstract}

Keywords Graphene, closed edges, nanopores, HRTEM, high temperature.

Graphene nanopores have been extensively studied, both experimentally and theoretically, primarily for application in emerging technologies for single-ion channels, ${ }^{1}$ single-molecule detection, ${ }^{2}$ and liquid purification..$^{3-7}$ The experimental sequencing of DNA using graphene technology is also 
rapidly advancing. ${ }^{8-10}$ Folded and non-folded DNA molecules can be distinguished by their difference in conductance when passed through graphene nanopores. Moreover, theoretical studies suggest that the conformation of DNA nucleotides in a graphene nanopore can be controlled through precise engineering of the nanopore surface. In consequence, strand sequencing of DNA by measuring the ionic current blockade in graphene nanopores is possible, enabling fast and inexpensive DNA sequencing technology. ${ }^{11-14}$

Due to these important potential applications, the introduction of a nanopore into a graphene sheet in a controlled style has been widely studied. ${ }^{15,16}$ Helium ion drilling, electron-beam lithography, focused electron beam irradiation, block copolymer lithography, defect-selective electron recoil sputtering, focused ion beam direct writing are among many approaches that have been applied to the creation and size control of graphene nanopores. ${ }^{17-20,16,21,22}$ However, small nanopores are often back-filled with both carbon and foreign atoms in a self-healing process due to the dangling bonds at the edge of the nanopore, ${ }^{23-26}$ and this instability is detrimental to the functionality and lifetime of the nanopores. Hence the development of approaches to prevent the filling of graphene holes after creation is a crucial step to improving their lifetime and consequent application in solution environments. One way to do this is to eliminate the highly reactive dangling bonds associated with the edges of graphene holes. A previous report has utilized Si atoms to stabilize graphene nanopores by bridging the dangling bonds around the perimeter of the hole. ${ }^{15}$ However functionalization of graphene edges still results in polar functional groups that are attractive to ionic species in solution. Elimination of polar or dangling bonds could be achieved by forming closed edge pores in bilayer graphene, similar to the edge of a carbon nanotube or a back folded graphene layer, where all C-C bonds are $\mathrm{sp}^{2}$ in nature, but where their flexibility enables bending and curvature of the lattice and subsequently sealing of the edges.

The utilization of electron beams within a transmission electron microscope for drilling holes into multi-layered graphene is well established. ${ }^{18,27-31}$ The additional use of an in-situ heating holder can also be applied to control the deposition of amorphous carbon.$^{29,31}$ To selectively remove the layers in multi-layered graphene, a top-down approach can be applied in which an electron beam at 
an energy beyond the graphene sputtering threshold voltage $(>80 \mathrm{keV})$ is focused onto an area of interest to decrease the number of layers, eventually leading to the formation of nanopores of desired size. ${ }^{28,30}$ However, the nature of electron beam sputtering is such that the back layer of a multilayered graphene sheet is eroded first. ${ }^{32}$ This leads to the back layer having a larger pore size and therefore the holes in each layer do not have aligned edges and it is hence difficult for the edges in adjacent layers to bond forming a closed edge nanopore.

To overcome this challenge in forming closed edge nanopores within monolayer graphene, we have exploited hydrocarbon surface adsorbates that are known to condense on graphene at room temperature. We show that a combination of controlled electron beam irradiation and variable of temperature can lead to stable closed edge nanopores in graphene. Using an in-situ heating holder has enabled us to study the structural changes of the same atoms between room temperature to $800^{\circ} \mathrm{C}$. This ability to track the same group of atoms reveals the detailed transformation of a nanopore and consequently demonstrates control over the size of the closed edge nanopores.

\section{Results and Discussion}

Open or closed edges and folded edges, has been extensively studied in graphene. ${ }^{33-36}$ The schematic model shown in figure $1(a, b)$ shows bilayer graphene in either its intrinsic open edge state or in a closed edge form with bonds between the upper and lower layer that saturate the dangling bonds. Monolayer graphene is typically covered with a surface layer of hydrocarbons at room temperature, forming a pseudo-bilayer material, in which one layer is amorphous. Heating to $\sim 500^{\circ} \mathrm{C}$ removes surface adsorbates to leave clean graphene. Understanding these temperature dependent surface chemistry interactions has enabled us to develop a process for converting surface adsorbates into a stable secondary layer that includes edge bonds suitable for closed nanopore formation. This process is schematically illustrated in figure $1 \mathrm{c}$. The graphene sample was initially heated to $800^{\circ} \mathrm{C}$ to allow surface contamination to evaporate, leaving clean graphene for which the lattice structure could be 
imaged.(Figure S2a in supporting information) Using a previously reported fabrication technique,$^{37,38}$ the electron beam $(80 \mathrm{keV})$ was focused to maximize the beam current density $\left(\sim 10^{8} \mathrm{e}^{-}\right.$ $\left.{ }^{1} \mathrm{~nm}^{-2} \mathrm{~s}^{-1}\right) ;{ }^{38}$ a nanopore was created as shown in process 1 , figure $1 \mathrm{c}$. The sample was then cooled to room temperature $(\mathrm{RT})$, process 2 , figure 1c which results in hydrocarbons from the microscope environment accumulating on the surface of the graphene. The rapid accumulation of the hydrocarbon residues has the effect of surrounding the hole and partially filling some of the nanopore. Exposure to the electron beam for extended periods at room temperature causes the nanopore to be completely filled. Electron beam irradiation of the hydrocarbon residue causes interlayer bonding to the underlying graphene layer, as shown in process 3 and 4 , figure $1 \mathrm{c}$, in addition to sputtering of hydrogen from the adsorbates to leave primarily carbon atoms, together with some small amounts of residual elements such as nitrogen and oxygen. Reheating the sample to $800^{\circ} \mathrm{C}$ causes the bound surface carbons to crystallize forming a polycrystalline secondary layer. The carbon atoms at the edge of the nanopore consequently bond to eliminate the dangling bonds (process 5). For this temperature cycle applied to the sample, the nanopore structure is stable against further temperature and vacuum level changes as the added layer does not evaporate due to electron beam irradiation. This is evident in figure S1 (supporting information), which shows only areas (at $800^{\circ} \mathrm{C}$ ) that have been exposed to extended electron beam irradiation have kept added layers whereas carbon sources on the surface of other areas vanish as the temperature increased from RT to $800^{\circ} \mathrm{C}$. Similarly, the treated graphene nanopore can now be reduced to RT without being backfilled. 


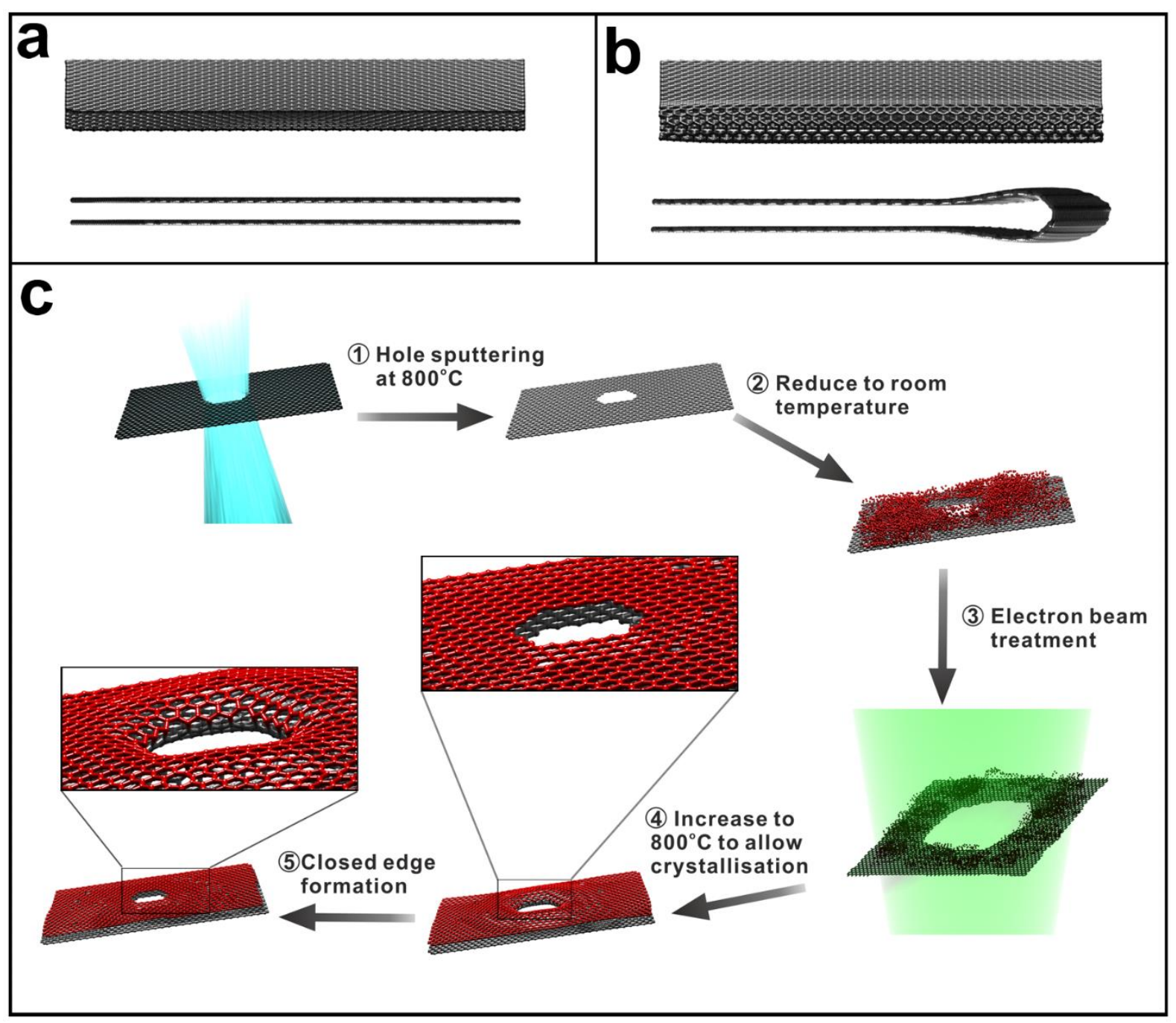

Figure 1. Closed edge graphene nanopore fabrication. (a, b) Atomic models of open and closed graphene edges (3D perspective front and side views). (c) Schematic illustration of temperature controlled formation of a stable closed edge graphene nanopore.

Phase contrast aberration-corrected transmission electron microscopy (AC-TEM) images can be used to distinguish differences between open and closed edges, based on their contrast profiles. We have compared multi-slice image simulations of both atomic models with experimental data. The details of the image processing techniques employed for all images are shown in figure S2 in supporting information. Atomic models of a nanopore in bi-layer graphene with both open and closed edges are shown in figures 2 a,b and 2 e,f respectively, and were used to generate the multislice image simulations in figure $2 \mathrm{c}$ and $2 \mathrm{~g}$. Simulated images of the open and closed edges (figures $2 \mathrm{~d}$ and $\mathrm{h}$ ) and analysis of these (figures $2 \mathrm{i}$ and $2 \mathrm{j}$ ) show that the closed edge gives rise to stronger contrast than the open edge relative to the contrast in the bulk lattice. The ratio between the 
intensities of the outermost atoms to bulk atoms is 1.17 for the closed edge and 1.06 for the open edge. A further example of several graphene nanopores which showed the closed edge formation process is shown in figure $2 \mathrm{k}$. This edge structure is similar to the image simulation of a closed edge nanopore. Analysis of a closed edge nanoribbon in figure S3 in the supporting information provides further evidence that bilayer graphene forms closed edges.
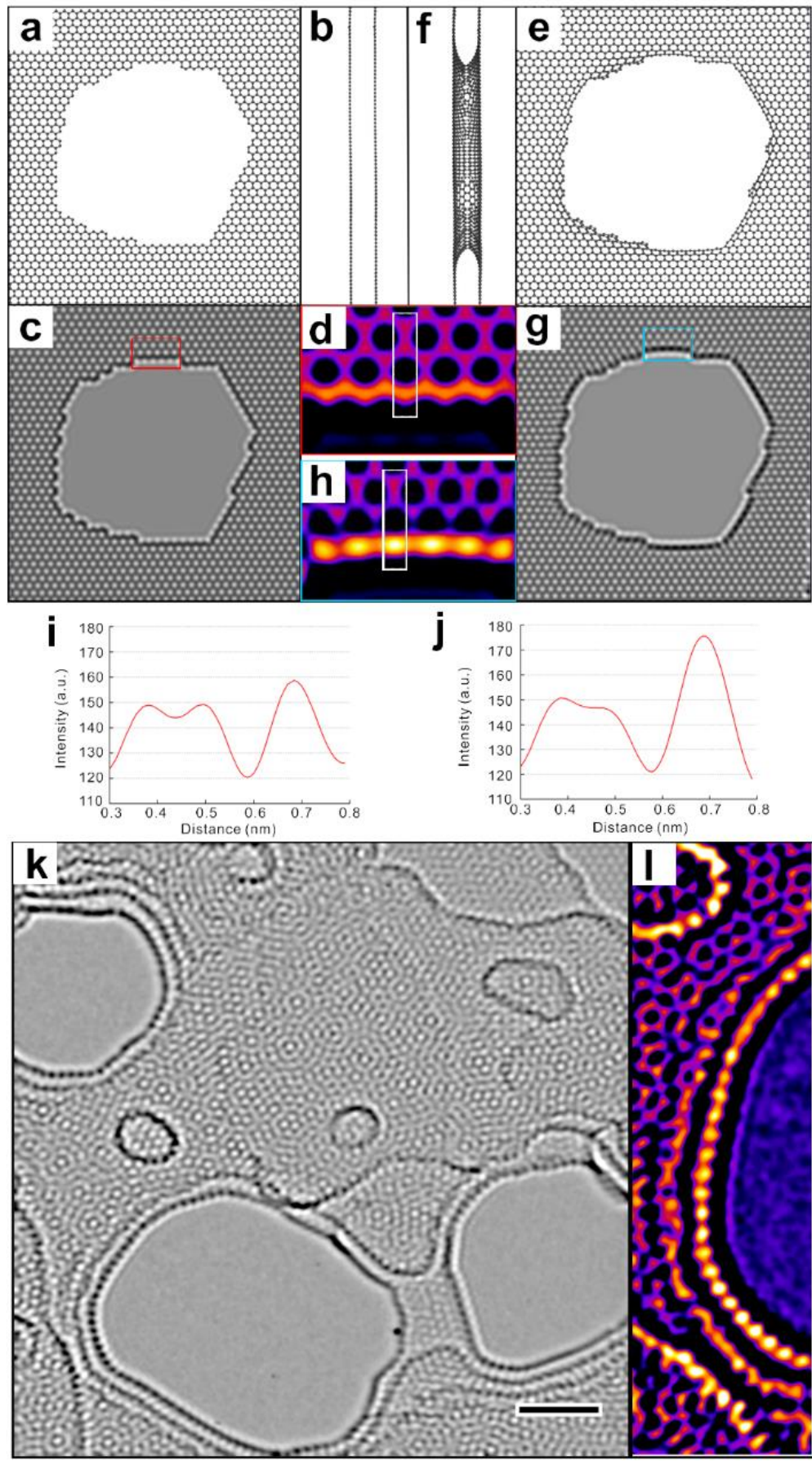

Figure 2. Modelling and simulation of open and closed edge graphene nanopores. $(a, b)$ Front and side views of an open edged graphene nanopore. (c) Multi-slice image simulation of the model in (a) with the 
region in the red rectangle enlarged in (d). The same analysis applied to a closed edge nanopore, shown in (e - h). (i) and (j) box profiles taken from the white rectangular regions in (d) and (h). (k) AC-TEM image of graphene nanopores with closed edges, magnified in (1). Scale bar $=2 \mathrm{~nm}$.

Figure 3 shows AC-TEM images of the same area during consecutive stages in the closed-edge nanopore formation process (schematically outlined in figure 1c). A $4 \mathrm{~nm}$ diameter nanopore was sputtered from pristine graphene sheet at a temperature of $800^{\circ} \mathrm{C}$ as shown in process 1 in figure 3 . The temperature was then reduced to RT (process 2) and the residual hydrocarbons were allowed to aggregate on the surface of graphene around the nanopore area, followed by further electron beam irradiation. The temperature was increased back to $800^{\circ} \mathrm{C}$ to promote crystallization and the construction of a closed edge (process 3). Finally the sample temperature was lowered to RT (process 4) showing that the structure of the closed edge is preserved at the probable working temperature for nanopore applications. Visual comparison between the nanopore edge after process 4 with that after process 2 shows significantly stronger edge contrast, typical of closed edge formation.
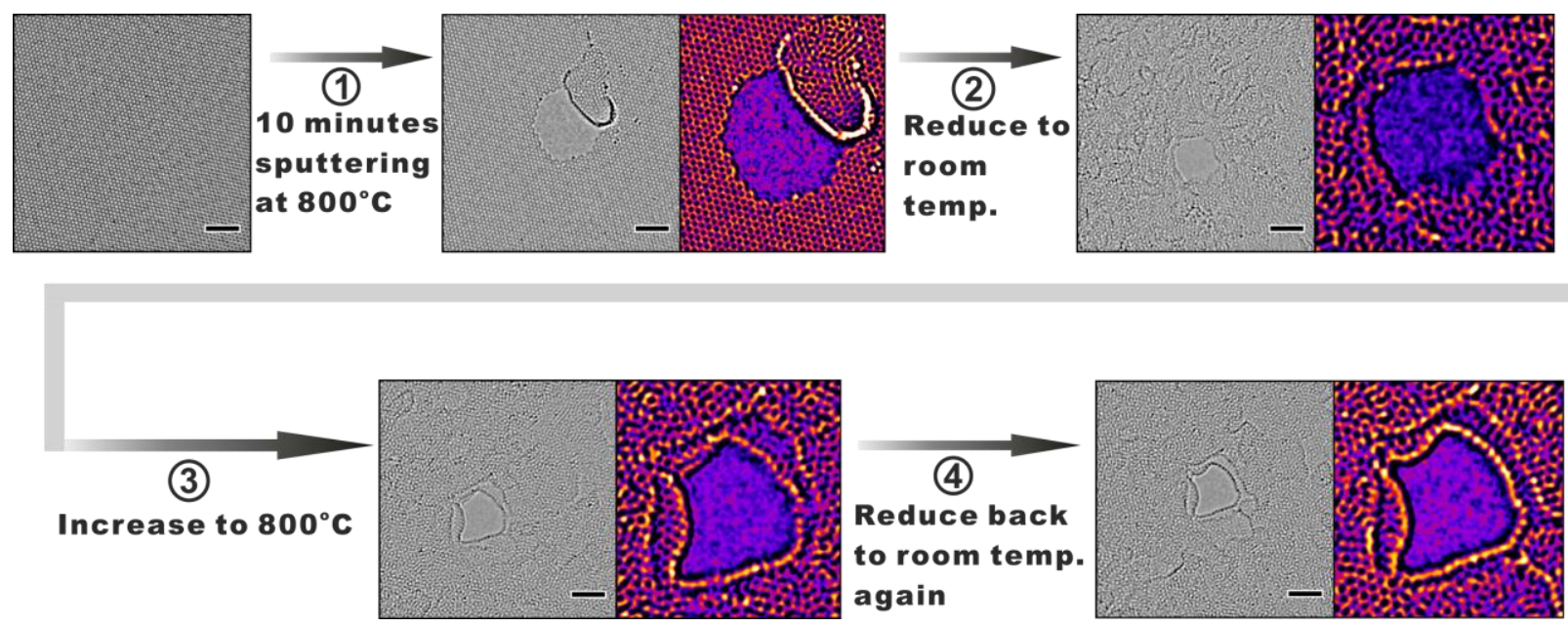

Figure 3. AC-TEM images showing the closed edge graphene nanopore experimental fabrication process. All scale bars $=2 \mathrm{~nm}$

Figure 4 provides more details that show how the surface hydrocarbons transform from amorphous to nanocrystalline $\mathrm{sp}^{2}$ regions as the temperature is increased. A nanopore was again initially sputtered at $800^{\circ} \mathrm{C}$ (figure 4a). The temperature was then reduced allowing amorphous hydrocarbons 
to condense on the surface as shown in figure $4 b$, and irradiated with the electron beam. The temperature was then increased progressively to monitor the structural evolution of the nanopore. The accumulated hydrocarbons were present in an amorphous state at both $\mathrm{RT}$ and $400^{\circ} \mathrm{C}$. However as the temperature was increased from 500 to $700^{\circ} \mathrm{C}$ (figures $4 \mathrm{~d}-\mathrm{f}$ ), the surface carbon starts to crystallize. This is evident from the power spectra shown in figures $4 \mathrm{~g}$ and i calculated from the highlighted boxed regions in figures $4 \mathrm{~b}$ and $\mathrm{f}$ respectively. The box profile across the center region of the power spectrum (figure $4 \mathrm{j}$ ) shows two peaks $(\mathrm{d}=0.21 \mathrm{~nm})$ and is characteristic of a polycrystalline $\mathrm{sp}^{2}$ lattice which is also evident from the Moiré patterns in figure $4 \mathrm{f}$. Figure $4 \mathrm{~g}$ has a relatively uniform intensity across the center of the power spectrum (figure $4 \mathrm{~h}$ ) indicative of an amorphous region. In figure $4 \mathrm{f}$, it appears that more than one layer has formed from the crystallization of the surface adsorbates, but the nanopore still has a closed-edge structure.
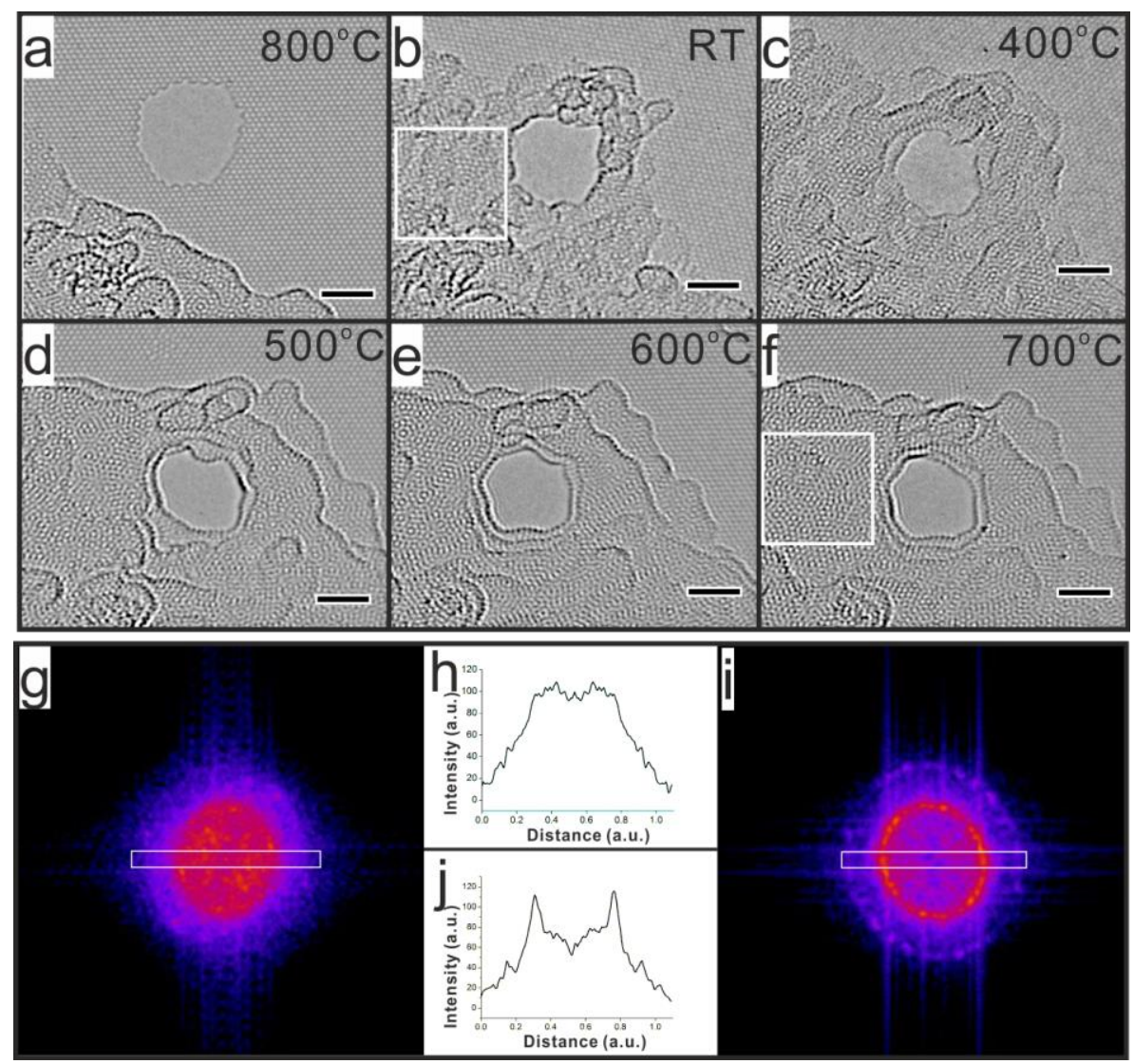

Figure 4. Temperature dependent study of graphene nanopores. $(a-f)$ AC-TEM images of the same graphene nanopore at various temperatures indicated. (g) and (i) power spectra calculated from the 
highlighted region of (b) and (f) respectively. (h) and (j) profiles from the white boxed region in (g) and (i). All scale bars $=2 \mathrm{~nm}$.

Improving the stability of the nanopores is the primary objective of this study and we have examined the stability of closed edge graphene nanopores at RT under electron beam irradiation and for a longer period of time without electron beam irradiation. To achieve this a nanopore was created at $800^{\circ} \mathrm{C}$ (figure 5a) and subsequently reduced to RT (figure 5b). When the temperature was increased back to $800^{\circ} \mathrm{C}$ a closed edge nanopore was created. The temperature was then finally reduced to RT and a sequence images recorded to monitor the nanopore stability. A total of 14 frames were recorded over 11 minutes and a selection of these are shown in figures $5 \mathrm{~d}-\mathrm{f}$ with the comprehensive data set provided in figure S4 in supporting information. The insets (Figures 5 c-f) are magnified images from the white boxed regions and the periodic bright features at the edge strongly suggest that these are closed edges. The area of the nanopore in successive frames has been measured and is plotted against time in figure $5 \mathrm{~g}$. The values fluctuate at around $15 \mathrm{~nm}^{2}$ with no significant decrease or increase over the course of our observations. The nanopore perimeters in the 14 frames recorded are shown superimposed in figure $5 \mathrm{~h}$ and no significant deviation from the initial shape is observed. This data indicates that once closed edges are formed, the dangling bonds diminish and edge stability is consequently increased. This process is effective in preventing the ascreated nanopore from backfilling by ambient carbon sources. The closed edge nanopores have also been tested for stability over an extended period of time. As shown in figure S5 in supporting information, six nanopores were initially created at $800^{\circ} \mathrm{C}$ and the previously described closed edge fabrication process was applied. After two days of exposure to atmospheric conditions, the asfabricated nanopores and their closed edges were intact and show no signs of clogging. Figures $5 \mathrm{i}$ and $\mathrm{j}$, respectively, show the same nanopore directly after fabrication and then after 2 days of exposure to the atmosphere outside the TEM. The closed edge nanopore has barely changed after showing that they are extremely robust to exposure to ambient conditions. It was important that the same nanopore was observed both before and after exposure to atmosphere for two days as the imaging process in the TEM requires electron beam irradiation which can create new holes in the 
sample that could be mistaken for the nanopores initially fabricated. However, since we recorded the exact location of the initial nanopore created we are confident that the nanopore shown in figure $5 \mathrm{j}$ is the same as that in figure 5i. Constant electron beam irradiation of the closed-edge nanopores also had little effect on their size once they had formed, indicating that the edges are robust against sputtering due their closed nature. ${ }^{39}$ In contrast, open edge holes are known to be continually sputtered and increase in size with exposure to the electron beam.

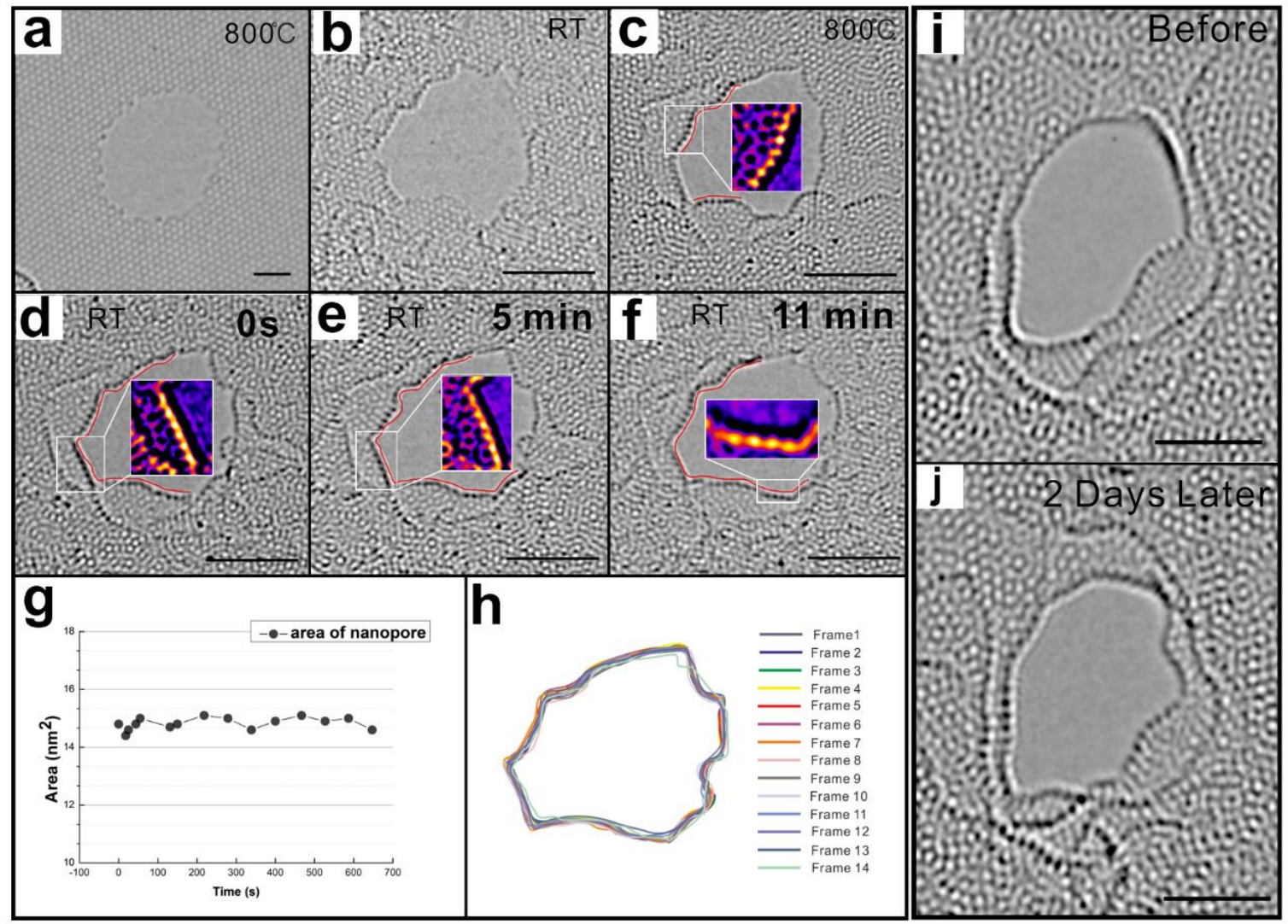

Figure 5. Graphene nanopore stability at room temperature. (a) Graphene nanopore in single layer graphene produced at $800^{\circ} \mathrm{C}$. (b) The same pore imaged after the temperature was reduced to RT. (c) Increasing the temperature back to $800^{\circ} \mathrm{C}$ enables the formation of closed edges. The temperature was then returned to RT for a second time and time series of images were recorded over 11 minutes $(\mathrm{d}-\mathrm{f})$. $(\mathrm{g}) \mathrm{The}$ area of the nanopore during the time series. (h) Perimeter of the nanopore during the time series using 14 frames in total illustrating the stability of the nanopore shape at room temperature under electron beam illumination. (i) Nanopore directly after fabrication and before exposure to atmosphere. (j) The same nanopore as in (i), but after two days of exposure to atmospheric conditions (outside the TEM). All scale bars $=2 \mathrm{~nm}$. 
The ability to control nanopore size is important as it affects the size of molecules that can pass. We demonstrate in figure 6 that the size of the nanopore could be adjusted during both the initial sputtering fabrication step at $800^{\circ} \mathrm{C}$ and the carbon source aggregation process at RT (process 2 in figure 3). Figures 6 (a-d) show four selective AC-TEM frames that form part of the graph shown in figure $6 \mathrm{q}$ at $800^{\circ} \mathrm{C}$. In this sequence electron beam irradiation strips off carbon atoms at the edge of the nanopore and results in an increased size with a growth rate of $0.01 \mathrm{~nm}^{2} / \mathrm{s}$ for the beam current density used. This is a relatively slow process and the average size of the nanopore at this stage could therefore be controlled with nanometer precision. When the temperature is reduced to RT, hydrocarbons adhere and slowly start to fill the nanopore and decrease its area linearly with time as shown in figure 6r. Four frames shown in figures $6 \mathrm{e}-\mathrm{h}$ demonstrate the appearance of the nanopore during this process with complete dataset shown in figure S6 in supporting information. The area of the nanopore decreased from $\sim 5.2 \mathrm{~nm}^{2}$ to $\sim 3.0 \mathrm{~nm}^{2}$ over 10 minutes, at a rate of $0.004 \mathrm{~nm}^{2} / \mathrm{s}$ (figure 6r). This gradual decrease also allows adjustment of the size of the nanopore with nanometer precision during this process. When the desired nanopore size is reached the temperature was raised to $800^{\circ} \mathrm{C}$ to fix the nanopore size and initiate closed edge formation, which limits any further size modification. Using the techniques described here, closed edge nanopores with sizes ranging from $\sim 1.5 \mathrm{~nm}^{2}$ to $\sim 70 \mathrm{~nm}^{2}$ were constructed using controlled exposure to the e-beam to determine the initial hole size followed by fixed time at RT to control the degree of hole shrinking by carbon adsorbate filling (figures 6i-p). Figure S8 shows that back filling from carbon condensation at low temperature $\left(200^{\circ} \mathrm{C}\right)$ occurs when the edges of nanopores are left open which illustrates the significance of closed edges in stabilizing nanopores for practical applications. 

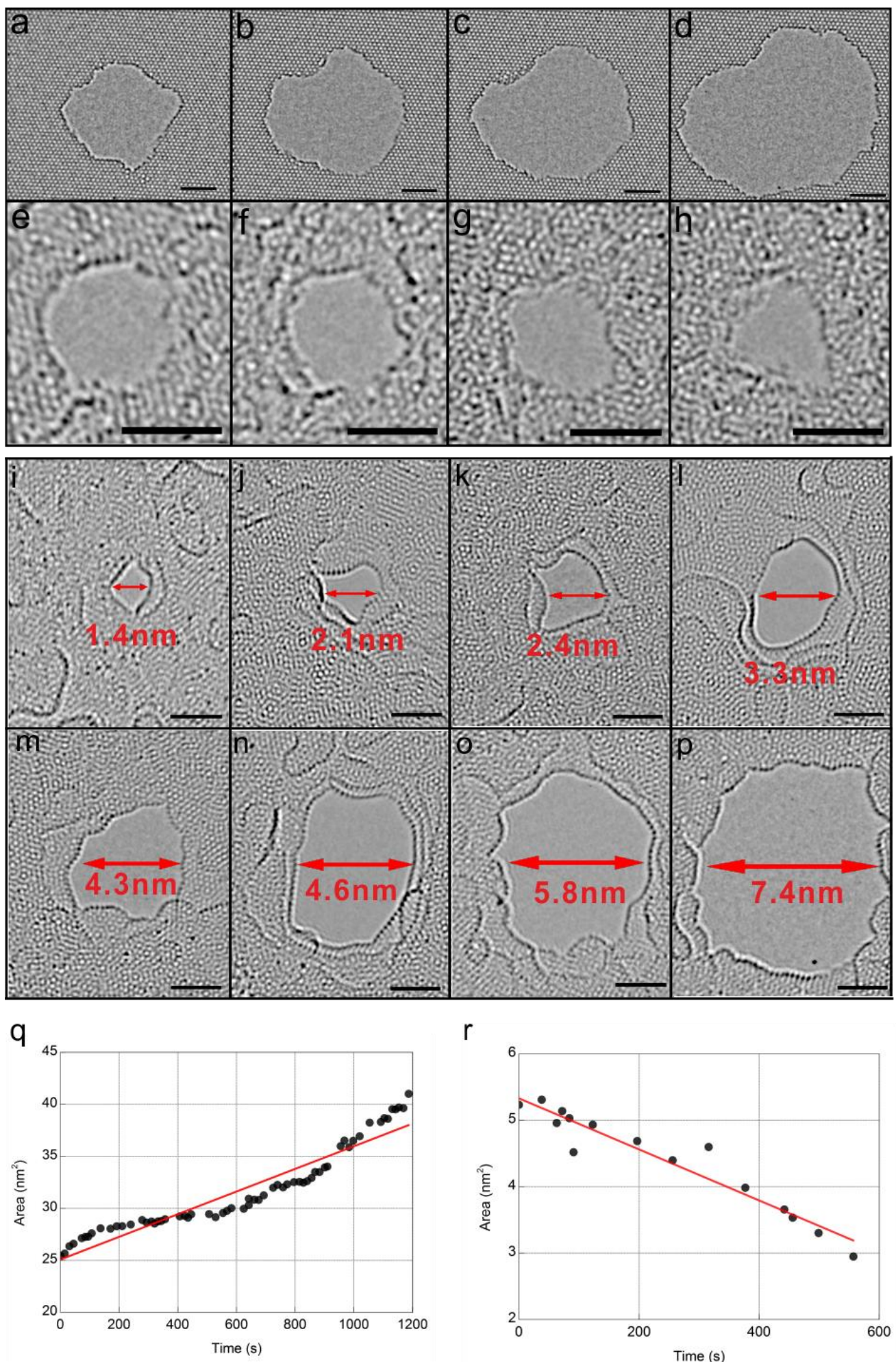

Figure 6. Nanopore size control. Nanopore sizes controlled during two separate stages; electron beam sputtering at $800^{\circ} \mathrm{C}$ and nanopore shrinkage at room temperature. $(\mathrm{a}-\mathrm{d})$ four selective frames from hole sputtering at $800^{\circ} \mathrm{C}$ with the area trend plotted in $(\mathrm{q})$ together with a linear fit. $(\mathrm{e}-\mathrm{h})$ four selective frames from room temperature nanopore shrinkage with the area trend shown in (r) together with a linear fit. Utilizing these two control parameters closed edge nanopores with diameters ranging from $1.4 \mathrm{~nm}$ to $7.4 \mathrm{~nm}$ were fabricated in a controlled manner as shown in $(\mathrm{i}-\mathrm{p})$. All scale bars are $=2 \mathrm{~nm}$. 


\section{Conclusion:}

Using a stable SiN based in-situ TEM heating chip that exhibits minimal drift, we have been able to control accurately the temperature of a graphene specimen and to monitor its effect on edge structures in graphene nanopores. This has enabled the development of a series of temperature assisted processes that form closed edges in graphene nanopores. The closed edge nanopores were stable during 11 minutes of observation and further experiments showed that they also stable for up to 2 days in air. The size of the nanopore can be controlled with nanometer precision by adjusting the electron beam irradiation time at $800^{\circ} \mathrm{C}$ during the fabrication process and the time spent at $\mathrm{RT}$ where additional carbon layers are added. This practical method of fabricating closed edges around graphene nanopores could address issues arising from the uncontrolled back filling of nanopores, leading to, for example, instability during DNA and single molecule detection using graphene nanopores.

\section{Methods:}

CVD growth of graphene on liquid copper. The graphene used in this experiment was synthesized by atmospheric pressure CVD. Copper foil (Alfa Aesar, puratonic 99.999\% purity, $0.1 \mathrm{~mm}$ thick) of $\sim 1 \mathrm{~cm}^{2}$ was used as a catalyst, resting on top of a similar-sized piece of tungsten (Alfa Aesar, 99.95\% purity, $0.1 \mathrm{~mm}$ thick). This was loaded into a quartz tube in a split-tube furnace CVD system, which was subsequently sealed and tested with a vacuum pump. The tube was then flushed with $200 \mathrm{sccm}$ Ar, $100 \mathrm{sccm} \mathrm{H} / \mathrm{Ar} \operatorname{mix}\left(25 \% \mathrm{H}_{2}\right)$ and $100 \mathrm{sccm} \mathrm{CH} \mathrm{CH}_{4} / \mathrm{Ar} \operatorname{mix}\left(1 \% \mathrm{CH}_{4}\right)$ gas flow for 30 minutes at room temperature. The catalyst was then annealed for 30 minutes at $1100^{\circ} \mathrm{C}$ under $200 \mathrm{sccm} \mathrm{Ar}$ and $100 \mathrm{sccm} \mathrm{H}_{2} / \mathrm{Ar}$ mixed gas flow, melting the copper. The $\mathrm{H}_{2} / \mathrm{Ar}$ mix flow was reduced to $80 \mathrm{sccm}$ and the $\mathrm{CH}_{4} / \mathrm{Ar}$ mix was set at $10 \mathrm{sccm}$ for 90 minutes at the same temperature. Finally, the sample was removed from the heating region of the furnace and rapidly cooled at ambient temperature under a $\mathrm{H}_{2}$ and $\mathrm{Ar}$ atmosphere. 
Transfer onto TEM grid. A (8\%wt. in anisole, 495 molecular weight) PMMA film was spin coated onto the graphene side of the sample at 4500 r.p.m for $60 \mathrm{~s}$, and then cured at $180^{\circ} \mathrm{C}$ for 90 s. The underlying copper were etched from the side by floating the sample on iron (III) chloride + hydrochloric acid solution for 3 days, until only a transparent PMMA/graphene film remained suspended on the surface. This was then cleaned by floating onto fresh DI water and $30 \%$ hydrochloric acid respectively and then cleaned again in DI water. The film was then transferred onto a SiN TEM grid designed for in-situ Transmission Electron Microscopy in a heating holder (DENSsolutions single tilt $30^{\circ}$ fitted with DENSsolutions High Temperature EM heater chip with a maximum operating temperature up to $800^{\circ} \mathrm{C}$ ). The thin $\mathrm{SiN}$ membrane on the heating holder contained several windows (size $3 \times 0.2 \mu \mathrm{m}$ ) produced using a Zeiss NVision SEM:FIB prior to graphene transfer. These windows were essential to enable HRTEM imaging of the graphene lattice without contrast from the SiN membrane. After drying in air for about 3 hours, the sample was baked on a hot plate for 15 minutes to remove water and to improve sample adhesion to the heater chip. The sample was then placed in a furnace at $350^{\circ} \mathrm{C}$ overnight to remove the PMMA.

High Resolution Transmission Electron Microscopy. HRTEM imaging was performed using the Oxford-JEOL JEM 2200MCO field-emission gun transmission electron microscope, fitted with CEOS probe and image aberration correctors and a double Wien Filter monochromator ${ }^{40}$ operated with a $5 \mu \mathrm{m}$ slit to reduce the energy spread of the electron beam to $217 \mathrm{meV}$ at an accelerating voltage of $80 \mathrm{kV}$. Images were recorded using a Gatan Ultrascan $4 \mathrm{k}$ x $4 \mathrm{k}$ CCD camera with $2 \mathrm{~s}$ acquisition times. The sample height was continuously adjusted using a piezo controller in the microscope goniometer to maintain a defocus value of $\sim 1 \mathrm{~nm}$. Nanopores were initially created in graphene using a focused electron beam with a beam current density (BCD) of $\sim 1 \mathrm{X} 10^{8}$ e.s $\mathrm{s}^{-1} \mathrm{~nm}^{-2}$ with 300s exposure time. Once a small nanopore was created the beam was expanded to give a reduced $\mathrm{BCD}$ of $\sim 0.1-1 \times 10^{6}$ e.s $^{-1} \mathrm{~nm}^{-2}$ for image acquisition.

Image Processing. Image processing was performed using the Image $\mathbf{J}$ software. First, a band-pass filter with a range between 100 to 1 pixels was applied to the Fourier Transform of the image to remove broad, uneven illumination variations. The image was then Gaussian smoothed (sigma value 
- 3) to remove further noise. A colour look-up table (LUT) 'Fire' in ImageJ was used to improve visual appearance. At all stages care was taken to ensure that these processes did not introduce artefacts in the final images. Multi-slice image simulations were performed using the JEMS software with appropriate supercells. The supercell structures were created using Accelrys Discovery Studio Visualizer. The detailed image processing techniques employed are shown in figure S2 in the supporting information.

\section{Acknowledgements}

JHW thanks the Royal Society for support. Financial support from the EU ESTEEM2 (Enabling Science and Technology through European Electron Microscopy) project ( $7^{\text {th }}$ Framework Programme of the European Commission) is acknowledged.

\section{Supporting Information Available.}

Low magnification images, image processing techniques employed, modelling and simulation of closed edge nanoribbon, comprehensive AC-TEM dataset, and supporting analysis are included in the supporting information. This material is available free of charge via the Internet at http://pubs.acs.org.

\section{References}

(1) Sint, K.; Wang, B.; Kra, P. Selective Ion Passage through Functionalized Graphene Nanopores. J. Am. Chem. Soc. 2008, 130, 16448-16449.

(2) Schedin, F.; Geim, A. K.; Morozov, S. V.; Hill, E. W.; Blake, P.; Katsnelson, M. I.; Novoselov, K. S. Detection of Individual Gas Molecules Adsorbed on Graphene. Nature Mater. 2007, 6, 652-655.

(3) Cohen-Tanugi, D.; Jeffrey C, G. Water Desalination across Nanoporous Graphene. Nano Letts. 2012, 12, 3602-3608.

(4) Suk, M. E.; Aluru, N. R. Water Transport through Ultrathin Graphene. J. Phys. Chem. Lett. 2010, 1, 1590-1594. 
(5) Cohen-Tanugi, D.; Grossman, J. C. Water Permeability of Nanoporous Graphene at Realistic Pressures for Reverse Osmosis Desalination. J. Chem. Phys. 2014, 131, 074704.

(6) Konatham, D.; Yu, J.; Ho, T. A.; Striolo, A. Simulation Insights for Graphene-Based Water Desalination Membranes. Langmuir 2013, 29, 11884-11897.

(7) Suk, M. E.; Aluru, N. R. Molecular and Continuum Hydrodynamics in Graphene Nanopores. RSC Adv. 2013, 3, 9365-9372.

(8) Schneider, G. F.; Kowalczyk, S. W.; Calado, V. E.; Pandraud, G.; Zandbergen, H. W.; Vandersypen, L. M. K.; Dekker, C. DNA Translocation through Graphene Nanopores. Nano Letts. 2010, 10, 3163-3167.

(9) Merchant, C. A.; Healy, K.; Wanunu, M.; Ray, V.; Peterman, N.; Bartel, J.; Fischbein, M. D.; Venta, K.; Luo, Z.; Johnson, A. T. C.; et al. DNA Translocation through Graphene Nanopores. Nano Letts. 2010, 10, 2915-2921.

(10) Min, S. K.; Kim, W. Y.; Cho, Y.; Kim, K. S. Fast DNA Sequencing with a Graphene-Based Nanochannel Device. Nature Nanotechnol. 2011, 6, 162-165.

(11) Nelson, T.; Zhang, B.; Prezhdo, O. V. Detection of Nucleic Acids with Graphene Nanopores: Ab Initio Characterization of a Novel Sequencing Device. Nano Letts. 2010, 10, 3237-3242.

(12) Postma, H. W. C. Rapid Sequencing of Individual DNA Molecules in Graphene Nanogaps. Nano Letts. 2010, 10, 420-425.

(13) Zhang, Z.; Shen, J.; Wang, H.; Wang, Q.; Zhang, J.; Liang, L. Effects of Graphene Nanopore Geometry on DNA Sequencing. J. Phys. Chem. Lett. 2014, 5, 1602-1607.

(14) Wells, D. B.; Belkin, M.; Comer, J.; Aksimentiev, A. Assessing Graphene Nanopores for Sequencing DNA. Nano Letts. 2012, 12, 4117-4123.

(15) Lee, J.; Yang, Z.; Zhou, W.; Pennycook, S. J.; Pantelides, S. T.; Chisholm, M. F. Stabilization of Graphene Nanopore. Proc. Natl. Acad. Sci. 2014, 111, 7522-7526.

(16) Krasheninnikov, A. V.; Banhart, F. Engineering of Nanostructured Carbon Materials with Electron or Ion Beams. Nature Mater. 2007, 6, 723-733.

(17) Kim, M.; Safron, N. S.; Han, E.; Arnold, M. S.; Gopalan, P. Fabrication and Characterization of Large-Area, Semiconducting Nanoperforated Graphene Materials. Nano Letts. 2010, 10, $1125-1131$.

(18) Russo, C. J.; Golovchenko, J. A. Atom-by-Atom Nucleation and Growth of Graphene Nanopores. Proc. Natl. Acad. Sci. 2012, 109, 5953-5957.

(19) Bell, D. C.; Lemme, M. C.; Stern, L. A.; Williams, J. R.; Marcus, C. M. Precision Cutting and Patterning of Graphene with Helium Ions. Nanotechnology 2009, 20, 455301.

(20) Bieri, M.; Treier, M.; Cai, J.; Aït-Mansour, K.; Ruffieux, P.; Gröning, O.; Gröning, P.; Kastler, M.; Rieger, R.; Feng, X.; et al. Porous Graphenes: Two-Dimensional Polymer Synthesis with Atomic Precision. Chem. Commun. 2009, 45, 6919-6921. 
(21) Lemme, M. C.; Bell, D. C.; Williams, J. R.; Stern, L. A.; Baugher, B. W. H.; Jarillo-Herrero, P.; Marcus, C. M. Etching of Graphene Devices with a Helium Ion Beam. ACS Nano 2009, 3, 2674-2676.

(22) Lehtinen, O.; Kotakoski, J.; Krasheninnikov, A. V; Keinonen, J. Cutting and Controlled Modification of Graphene with Ion Beams. Nanotechnology 2011, 22, 175306.

(23) Zan, R.; Ramasse, Q. M.; Bangert, U.; Novoselov, K. S. Graphene Reknits Its Holes. Nano Letts. 2012, 12, 3936-3940.

(24) Barreiro, A.; Börrnert, F.; Avdoshenko, S. M.; Rellinghaus, B.; Cuniberti, G.; Rümmeli, M. H.; Vandersypen, L. M. K. Understanding the Catalyst-Free Transformation of Amorphous Carbon into Graphene by Current-Induced Annealing. Sci. Rep. 2013, 3, 1115.

(25) Tsetseris, L.; Pantelides, S. T. Adatom Complexes and Self-Healing Mechanisms on Graphene and Single-Wall Carbon Nanotubes. Carbon 2009, 47, 901-908.

(26) Zhao, J.; Deng, Q.; Bachmatiuk, A.; Sandeep, G.; Popov, A.; Eckert, J.; Rümmeli, M. H. Free-Standing Single-Atom-Thick Iron Membranes Suspended in Graphene Pores. Science 2014, 343, 1228-1232.

(27) Fischbein, M. D.; Drndić, M. Electron Beam Nanosculpting of Suspended Graphene Sheets. Appl. Phys. Lett. 2008, 93, 113107.

(28) Liu, S.; Zhao, Q.; Xu, J.; Yan, K.; Peng, H.; Yang, F.; You, L.; Yu, D. Fast and Controllable Fabrication of Suspended Graphene Nanopore Devices. Nanotechnology 2012, 23, 085301.

(29) Lu, N.; Wang, J.; Floresca, H. C.; Kim, M. J. In Situ Studies on the Shrinkage and Expansion of Graphene Nanopores under Electron Beam Irradiation at Temperatures in the Range of 400-1200 C. Carbon 2012, 50, 2961-2965.

(30) Schneider, G. F.; Xu, Q.; Hage, S.; Luik, S.; Spoor, J. N. H.; Malladi, S.; Zandbergen, H.; Dekker, C. Tailoring the Hydrophobicity of Graphene for Its Use as Nanopores for DNA Translocation. Nature Commun. 2013, 4, 2619.

(31) Song, B.; Schneider, G. F.; Xu, Q.; Pandraud, G.; Dekker, C.; Zandbergen, H. Atomic-Scale Electron-Beam Sculpting of near-Defect-Free Graphene Nanostructures. Nano Letts. 2011, 11, 2247-2250.

(32) Warner, J. H.; Mukai, M.; Kirkland, A. I. Atomic Structure of ABC Rhombohedral Stacked Trilayer Graphene. ACS Nano 2012, 6, 5680-5686.

(33) Liu, Z.; Suenaga, K.; Harris, P.; Iijima, S. Open and Closed Edges of Graphene Layers. Phys. Rev. Lett. 2009, 102, 015501.

(34) Zhan, D.; Liu, L.; Xu, Y. N.; Ni, Z. H.; Yan, J. X.; Zhao, C.; Shen, Z. X. Low Temperature Edge Dynamics of AB-Stacked Bilayer Graphene: Naturally Favored Closed Zigzag Edges. Sci. Rep. 2011, 1, 12.

(35) Meyer, J. C.; Geim, A. K.; Katsnelson, M. I.; Novoselov, K. S.; Booth, T. J.; Roth, S. The Structure of Suspended Graphene Sheets. Nature 2007, 446, 60-63.

(36) Kim, K.; Lee, Z.; Malone, B. D.; Chan, K. T.; Alemán, B.; Regan, W.; Gannett, W.; Crommie, M. F.; Cohen, M. L.; Zettl, A. Multiply Folded Graphene. Phys. Rev. B 2011, 83, 245433. 
(37) He, K.; Lee, G.-D.; Robertson, A. W.; Yoon, E.; Warner, J. H. Hydrogen-Free Graphene Edges. Nature Commun. 2014, 5, 3040.

(38) Robertson, A. W.; Allen, C. S.; Wu, Y. A.; He, K.; Olivier, J.; Neethling, J.; Kirkland, A. I.; Warner, J. H. Spatial Control of Defect Creation in Graphene at the Nanoscale. Nature Commun. 2012, 3, 1144.

(39) Warner, J. H.; Rümmeli, M. H.; Bachmatiuk, A.; Büchner, B. Examining the Stability of Folded Graphene Edges against Electron Beam Induced Sputtering with Atomic Resolution. Nanotechnology 2010, 21, 325702.

(40) Mukai, M.; Kim, J. S.; Omoto, K.; Sawada, H.; Kimura, A.; Ikeda, A.; Zhou, J.; Kaneyama, T.; Young, N. P.; Warner, J. H.; et al. The Development of a $200 \mathrm{kV}$ Monochromated Field Emission Electron Source. Ultramicroscopy 2014, 140, 37-43.

\section{Table of Content graphic}

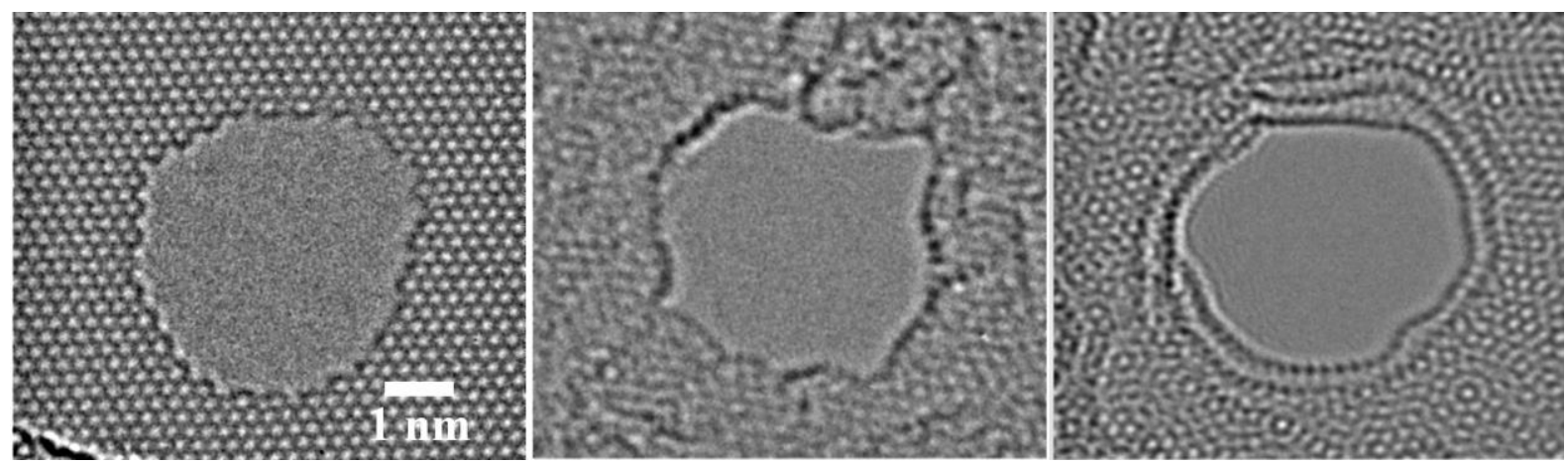

